\title{
PET/CT and contrast-enhanced CT: making a difference in assessment and staging of patients with lymphoma
}

\author{
Ashraf Anas Zytoon ${ }^{1 *}$ (D), Hala Hafez Mohamed', Basma Adel Abd Elaziz Mostafa² and \\ Mohamed Mohamed Houseni
}

\begin{abstract}
Background: The aim of this study is to evaluate the diagnostic performance of contrast-enhanced computed tomography (CECT) and 2-[Fluorine-18] fluoro-2-deoxy-D-glucose positron emission tomography combined to computed tomography (18 F-FDG PET/CT) in assessment of lymphoma.

Methods: Hundred patients, pathologically proven as lymphoma, were evaluated by CECT and 18F-FDG PET/CT for initial assessment and staging of the disease. The number of lesions and the disease stage detected by each modality was calculated and further analyzed to be compared.

Results: 18F-FDG PET/CT diagnosed a total number of 545 lymphoma involved regions with sensitivity 96.6\%, specificity 98.8\%, and accuracy 99\% that was higher than CECT which diagnosed a total number of 439 lymphomatous regions with sensitivity $87.5 \%$, specificity $85.7 \%$, and accuracy $88 \%$. Discordant staging by both modalities was found in $23 \%$ of the patients. Lymphoma was upstaged by PET/CT in 17\% of the patients; with major changes in 12\% of them and downstaged in $6 \%$ of the patients.

Conclusion: 18F-FDG PET/CT scan has a better diagnostic performance, represented by sensitivity, specificity, and accuracy, than CECT scan in the initial assessment of lymphoma regarding its nodal and extra-nodal lesions that could lead to alteration of disease staging which in turn markedly affecting the decision of treatment regimens.
\end{abstract}

Keywords: CECT: contrast-enhanced computed tomography, 18F-FDG PET/CT: 2-[Fluorine-18] fluoro-2-deoxy-D-glucose positron emission tomography combined to computed tomography

\section{Background}

Lymphomas are heterogeneous group of diseases that arise from the constituent cells of the immune system or from their precursors. They are known to arise from virtually any organ or tissue in the body. Lymphomas are broadly classified into two main groups: non-Hodgkin's lymphoma (NHL) and Hodgkin's lymphoma (HL) [1]. Accurate diagnosis, correct staging, and proper therapy are important for successful outcome [2]. Diagnostic imaging plays a critical role in the initial evaluation, monitoring, and follow-up of lymphoma patients [3].

\footnotetext{
*Correspondence: ashradio@gmail.com

${ }^{1}$ Radiodiagnosis Department, Faculty of Medicine, Menoufia University, Menoufia, Egypt

Full list of author information is available at the end of the article
}

Computed tomography (CT) used to be the imaging modality of choice for staging lymphoma because of its widespread availability and relatively low cost [1]. In combination with powered injectors for rapid bolus administration of intravenous contrast medium used in contrast-enhanced CT (CECT) scan, lymph nodes of 5 $\mathrm{mm}$ or less in diameter and extra-nodal lesions with altered enhancement can be identified [4] At initial staging of lymphoma, determination of nodal involvement by CECT is based on size criteria; if the short-axis diameter is more than $10 \mathrm{~mm}$ and/or the long-axis diameter of $15 \mathrm{~mm}$ are exceeded. General criteria for extra-nodal involvement are any focal density alterations, abnormal contrast enhancement, or mass lesions involving soft tissues, bones, parenchymal organs, or serosal cavities [5]. 
Lymphoma may be restricted to the lymphatic system and/or present as extra-nodal disease, so diverse imaging appearance is expected [6]. Extra nodal lymphomas, seen mainly in NHL, can involve any organ. Secondary extension from a disseminated disease is the most frequent, while isolated primary lesions are possible but rare [7]. Ann Arbor's staging system was commonly used for both Hodgkin's and non-Hodgkin's lymphoma but currently, the Lugano classification was developed to simplify and standardize staging and response assessment [2]. Positron emission tomography (PET) using 2-[fluorine-18] fluoro-2-deoxy-D glucose (FDG) has already been validated to assess patients with different types of malignant tumors, including lymphomas. The principle of the imaging test is based on metabolic changes that reflect fundamental differences in the central metabolic pathways in malignant tissue [8]. 18F-FDG is the most commonly used radiotracer in PET imaging. FDG is an analogue of glucose and the uptake is directly proportional to the glucose metabolism of tumor tissue. Malignant tumors with high glucose metabolism show preferential uptake of FDG as compared to surrounding normal cells [2]. The standardized uptake value (SUV) is a semiquantitative parameter that is widely used in PET analysis and is proportional to the rate of glucose metabolism within the normal range of serum glucose concentrations [9]. Although the metabolic information afforded by PET imaging is invaluable, the quality of obtained data is poor/ noisy and limits imaging spatial resolution due to lack of detailed anatomical information, which impedes precise localization of sites with FDG uptake. For this reason, PET scan is often combined with CT imaging, allowing correlation between functional and anatomical imaging [10]. PET is especially helpful in oncology to visualize metabolic processes in malignant tissues. Combined with X-ray computed tomography (CT), it substantially enhances the value of diagnostic results [11]. Treatment is based on a variable combination of chemotherapy, radiotherapy, and immunotherapy, adapted to each type [7].

\section{Methods}

The research methodology included the following items:

- Inclusion and exclusion criteria of the studied patients.

- Imaging protocol such as patients preparation, scanning technique and imaging parameters, post scanning instructions, image analysis and data interpretation, and diagnostic criteria of lymphoma by CECT and 18F-FDG PET/CT.

\section{Patients}

We retrospectively studied 100 lymphoma patients attended to the radiology department at our institute from December 2017 to July 2019 for initial assessment and staging of the disease by CECT and 18F-FDG PET/ CT scans. The study included adult patients; their ages ranged from 20 up to 70 years distributed as 61 males and 39 females with initial presentation of pathologically proven untreated lymphoma and excluded patients with other malignancies, renal failure, pregnancy, and hypersensitivity to contrast agents.

\section{Imaging method \\ Protocol}

All patients in the study were subjected to full history and complete clinical examination including the clinical stage of the disease. The patients were instructed to fast for $6 \mathrm{~h}$ before the scan. Creatinine clearance was checked to be $>30$ and blood glucose level was ensured to be below $200 \mathrm{mg} / \mathrm{dl}$ as non-insulindependent diabetic patients were allowed to take small meal with oral anti-diabetic medications then fasting for $6 \mathrm{~h}$ but insulin-dependent diabetic patients were allowed to take early breakfast with their normal insulin dose then fasting for $4 \mathrm{~h}$. On the day of scan, diabetic patients with blood glucose level higher than $200 \mathrm{mg} / \mathrm{dl}$ were injected rapid acting subcutaneous insulin and re-evaluated $2 \mathrm{~h}$ later. If controlled blood glucose level, they were scheduled late in the list to avoid the high FDG uptake by muscles and heart shortly after insulin injection but if not controlled blood glucose level, the patient was scheduled on another day.

\section{Scanning technique and imaging parameters}

Intravenous injection (IV) of 2-[fluorine-18] fluoro-2deoxy-D-glucose 18 F-FDG through IV line with a dose of $0.1 \mathrm{mCi} / \mathrm{kg}$ was done. After tracer injection, patient was asked to stay for $60-70 \mathrm{~min}$ in a quite dark room covered by warm blankets. No speaking, chewing, or reading was allowed. The patient was taken to the scan room where Siemens Biograph MCT 128 PET/CT exists, as its PET component from Knoxville, USA, and its CT component from Forchheim, Germany. First, a low-dose CT scan at $120 \mathrm{kV}, 100 \mathrm{mAs}, 0.9$ pitch, $5 \mathrm{~mm}$ slice thickness, for attenuation correction, was obtained at the range extending from skull base down to upper-thighs. This is immediately followed by a three-dimensional PET acquisition at $3 \mathrm{~min} / \mathrm{bed}$ position, $6-9$ bed positions/patient, field of view $11.4 \mathrm{~cm} /$ bed position; obtained at the same scan range. Iterative algorithms, TrueX+TOF ultra HD-PET, 2 iterations, 21 subsets, were used for reconstruction of the PET images. Data were filtered using FWHM $4.0 \mathrm{~mm}$ with 3D-Gaussian Kernel and corrected for scatter. After completion of the PET scan, contrast-enhanced CT at $120 \mathrm{kV}, 300 \mathrm{mAs}$, 0.9 pitch, $5 \mathrm{~mm}$ slice thickness, and $2.5 \mathrm{~mm}$ 
reconstruction thickness was done at the same scan range after IV injection of $1.5-2 \mathrm{ml} / \mathrm{kg}$ of an iodinated contrast material (Optray 300) using automated injector (Medrad Stellant) with flow rate $4 \mathrm{ml} / \mathrm{s}$. A limited breath-hold technique was used to avoid motioninduced artifacts. Additional maximum inspiration CT scan for detection of lung lesion was performed. The radiation dose was $8.19 \pm 0.83 \mathrm{mSv}$ and $13.44 \pm 5.14$ $\mathrm{mSv}$ for PET and CT components, respectively, resulting in a total dose of $21.64 \pm 5.20 \mathrm{mSv}$. That was in agreement with Gomez et al. [12] who mentioned that in 2008, in the USA, the diagnose technique that showed the highest dose deposition in the population was CT scans and Yuhao L [13].

\section{Post scanning instructions}

Although the level of radiation, emitting out of the patient after PET scan, is low, it is advised to avoid close contact with children and pregnant women and stop breast feeding for $24 \mathrm{~h}$. If traveling abroad within $48 \mathrm{~h}$ is planned, it may trigger the sensitive radiation detectors, so it is advised to have a document stating performing PET scan during the last $48 \mathrm{~h}$. Well hydration and frequent voiding are advised to help flush the radioactive material out of the body.

\section{Image analysis and data interpretation}

The attenuation-corrected FDG-PET images, CT lowdose images, as well as contrast-enhanced CT images were automatically fused on True D Siemens software or transferred to Philips Intellispace Portal and OSIRIX fusion workstation. Contrast-enhanced CT (CECT) images are evaluated at axial, coronal, and sagittal reconstructed planes. Maximum standardized uptake value (SUVmax) representing the quantitative analysis that measure the degree of FDG uptake at detected lesions was done by applying circular ROI with average diameter $2 \mathrm{~cm}$ over the most active part of the lesion. For each patient, 5 lymph node groups including cervical, axillary, mediastinal, abdominopelvic and inguinal groups, spleen, bone marrow, and other body tissues grouped in 9 extranodal sites including lung, liver, rest of GIT, rest of the chest, renoadrenal, muscles, head and neck, breast and skin with subcutaneous tissues were evaluated for lesions at CECT and PET/CT images. So, the total number of examined LN groups was 500 groups/100 patients as the group is considered involved even if a single LN of the group is affected. The spleen is considered involved whatever the number of its lesions, so the total number was 100 spleens/100 patients. The bone marrow was considered involved whatever the number of its lesions, so the total number is 100 bone marrows $/ 100$ patients. The other extra nodal lesions examined in 9 main organs as mentioned before, so the total number is 900 other extranodal organs $/ 100$ patients. Then staging (according Lugano classification) of lymphoma is done.

\section{Diagnostic criteria of lymphoma by CECT}

General criteria for lymph nodal involvement: if the short-axis diameter is more than $10 \mathrm{~mm}$ and/or the long-axis diameter of $15 \mathrm{~mm}$ are exceeded. General criteria for extra-nodal involvement are any focal density alterations, abnormal contrast enhancement, or mass lesions involving soft tissues, bones, parenchymal organs, or serosal cavities [5].

All lesions detected on CECT images were reevaluated on fused PET/CT images for estimation of their SUV max and correlation with FDG uptake by the mediastinal reference background.

\section{Diagnostic criteria of lymphoma by 18F-FDG PET/CT}

Any focus of elevated FDG uptake above mediastinal reference background whether on top of an obvious lesion on CECT or not [14].

Four readers in two teams interpreted the same series of patients, with blinding of the imaging reports and the other reviewers' interpretations. The first team is made up of two radiologists that interpret the CECT scan together. The second team is made up of two radiologists with experience in PET/CT reporting.

\section{Data analysis}

Data were statistically described in terms of number of lesions, percentages, mean \pm standard deviation $( \pm S D)$, and significance by $P$ value when appropriate; sensitivity, specificity, and accuracy of each modality were calculated and compared.

\section{Results}

\section{Demographic data}

Our study included 100 lymphoma patients where NHL represented $66 \%$ of the cases compared to $34 \%$ HL. In NHL, male affection (39\%) was higher than female affection (27\%). Also in HL, male affection (22\%) was higher than female affection (12\%). The most affected age group by NHL $24 \%$ was $50-<60$ years, while the most affected age group by HL $12 \%$ was $20-<30$ years (Table 1 ; Fig. 1).

\section{Contrast-enhanced computed tomography}

CECT detected 439 true positive affected sites and 32/ 471 false positive sites representing a percent of $6.8 \%$, as follows (Table 2; Fig. 2):

- Nodal involvement was truly positive in $266 \mathrm{LN}$ groups with 6 false positive results representing 
Table 1 Descriptive statistics of both lymphoma types

\begin{tabular}{|c|c|c|c|c|c|c|c|}
\hline \multicolumn{2}{|c|}{ Lymphoma type } & \multicolumn{2}{|l|}{$\mathrm{HL}$} & \multicolumn{2}{|l|}{ NHL } & \multicolumn{2}{|l|}{ Total } \\
\hline & & $\begin{array}{l}\text { Num } \\
34\end{array}$ & $\begin{array}{l}\% \\
34 \%\end{array}$ & $\begin{array}{l}\text { Num } \\
66\end{array}$ & $\begin{array}{l}\% \\
66 \%\end{array}$ & $\begin{array}{l}\text { Num } \\
100\end{array}$ & $\begin{array}{l}\% \\
100 \%\end{array}$ \\
\hline \multirow{5}{*}{$\begin{array}{l}\text { Age } \\
\text { groups }\end{array}$} & $20-<30$ years & 12 & $12 \%$ & 4 & $4 \%$ & 16 & $16 \%$ \\
\hline & $30-<40$ years & 9 & $9 \%$ & 7 & $7 \%$ & 16 & $16 \%$ \\
\hline & $40-<50$ years & 6 & $6 \%$ & 16 & $16 \%$ & 22 & $22 \%$ \\
\hline & $50-<60$ years & 3 & $3 \%$ & 24 & $24 \%$ & 27 & $27 \%$ \\
\hline & $60-70$ years & 4 & $4 \%$ & 15 & $15 \%$ & 19 & $19 \%$ \\
\hline \multirow[t]{2}{*}{ Sex } & Male & 22 & $22 \%$ & 39 & $39 \%$ & 61 & $61 \%$ \\
\hline & Female & 12 & $12 \%$ & 27 & $27 \%$ & 39 & $39 \%$ \\
\hline
\end{tabular}

reactive (inflammatory) lymph nodal enlargement and 46 LN groups were false negative.

- Splenic involvement was truly positive in 47 patients with 2 false positive results representing simple cysts. Also, there were 22 false negative results.

- Bone marrow involvement was truly positive in 41 patients with 12 false positive results representing benign lesions including hemangiomas, osteomyelitis, and degenerative changes with 13 false negative results.

- Other extranodal involvement was truly positive in 85 extranodal organs with 12 false positive results, representing hepatic cysts, breast fibroadenoma, thymic hyperplasia and adrenal adenoma with 25 false negative results.

- The false negative results in all examined sites were proved to be positive lesions by their increased FDG uptake on PET/CT.

\section{F-FDG PET/CT}

F-FDG PET/CT detected 545 true positive involved sites and 10/555 false positive sites representing a percent of $1.8 \%$, as follows (Table 3; Fig. 3):

- Nodal involvement was truly positive in $312 \mathrm{LN}$ groups with 4 false positive results due to inflammatory process correlated with clinical data and 3 false negative nodal results.

- Splenic involvement was truly positive in 79 patients with 1 false positive result representing splenic abscess correlated with clinical data with no false negative results.

- Bone marrow involvement was truly positive in 54 patients with 1 false positive result at the superolateral femoral neck discovered by magnetic resonance imaging (MRI) to be a stress fracture with no false negative results.

- Other extra nodal involvement was truly positive in 100 extranodal organs with 4 false positive results representing chronic tonsillitis and post-

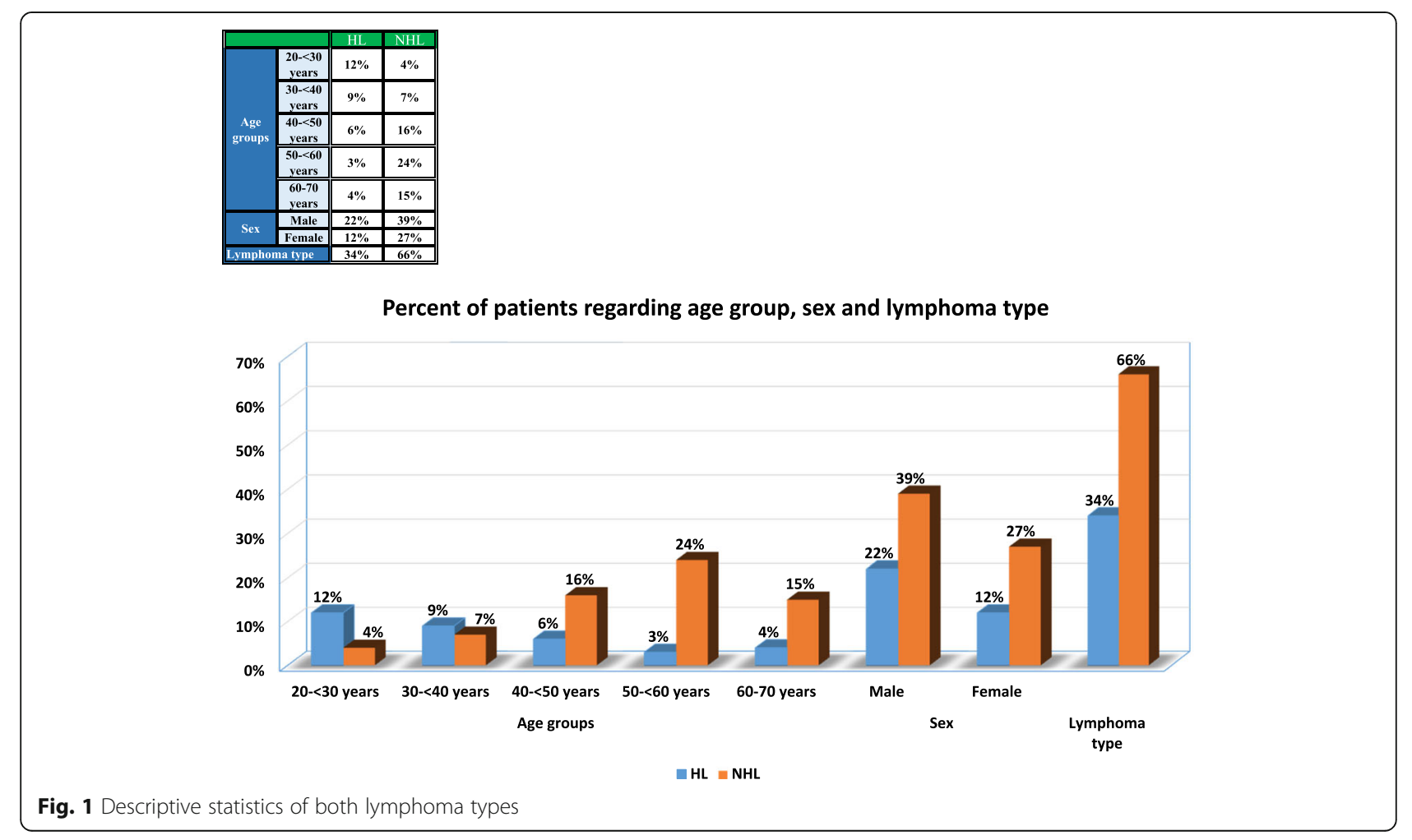


Table 2 Descriptive statistics of the number of lesions detected by contrast-enhanced computed tomography (CECT) scan

\begin{tabular}{llllll}
\hline Parameters & \multicolumn{4}{l}{ Number of lesions detected by CECT scan } & \\
\cline { 2 - 6 } & Lymph node groups & Spleen & Bone marrow & Other extra-nodal organs & Total \\
\hline True positive & 266 & 47 & 41 & 85 & 439 \\
False positive & 6 & 2 & 12 & 12 & 32 \\
False negative & 46 & 22 & 13 & 25 & 778 \\
True negative & 182 & 29 & 34 & 900 & 1023 \\
Total examined sites in 100 patients & 500 & 100 & 100 & 900 \\
\hline
\end{tabular}

biopsy subcutaneous axillary inflammatory changes correlated with clinical data with no false negative results.

Regarding the involved groups of lymph nodes by both CECT and 18 F-FDG PET/CT

PET/CT detected more affected LN groups than those detected by CECT with the same order of LN group's involvement by both modalities, as follows: the most common involved lymph node group was the abdomino-pelvic group by a percent of $74 \%$ by PET/ CT and $67 \%$ by CECT, then the mediastinal group by a percent of $73 \%$ by $\mathrm{PET} / \mathrm{CT}$ and $66 \%$ by CECT, followed by the cervical group by percent of $70 \%$ by PET/CT and $48 \%$ by CECT representing significant $P$ value, then the axillary group by percent of $51 \%$ by PET/CT and $44 \%$ by CECT and finally the inguinal group by percent of $45 \%$ by PET/CT and $40 \%$ by CECT (Table 4; Fig. 4).
Regarding the involved other extra nodal organs by $18 \mathrm{~F}$ FDG PET/CT

One hundred true positive extranodal organ involvements were detected. The most common involved extranodal organ was the lung 19\%, while the least common involved site was the muscles. The organs showing the highest SUV max by PET/CT were lung 44.0, stomach 33.4, submandibular glands 32.7 , and kidney30.8, while the organs showing the lowest SUV max were thymus 2.80, pleura 5.50, peritoneum 6.50, and liver 7.00 (Table 5; Fig. 5).

\section{Regarding diagnostic performance represented by sensitivity, specificity, and accuracy of CECT and PET/CT scans}

- For total lesions detection: PET/CT showed higher sensitivity $96.6 \%$, specificity $98.8 \%$, and accuracy $99 \%$ than CECT sensitivity $87.5 \%$, specificity $85.7 \%$, and accuracy $88 \%$ respectively, as follows:

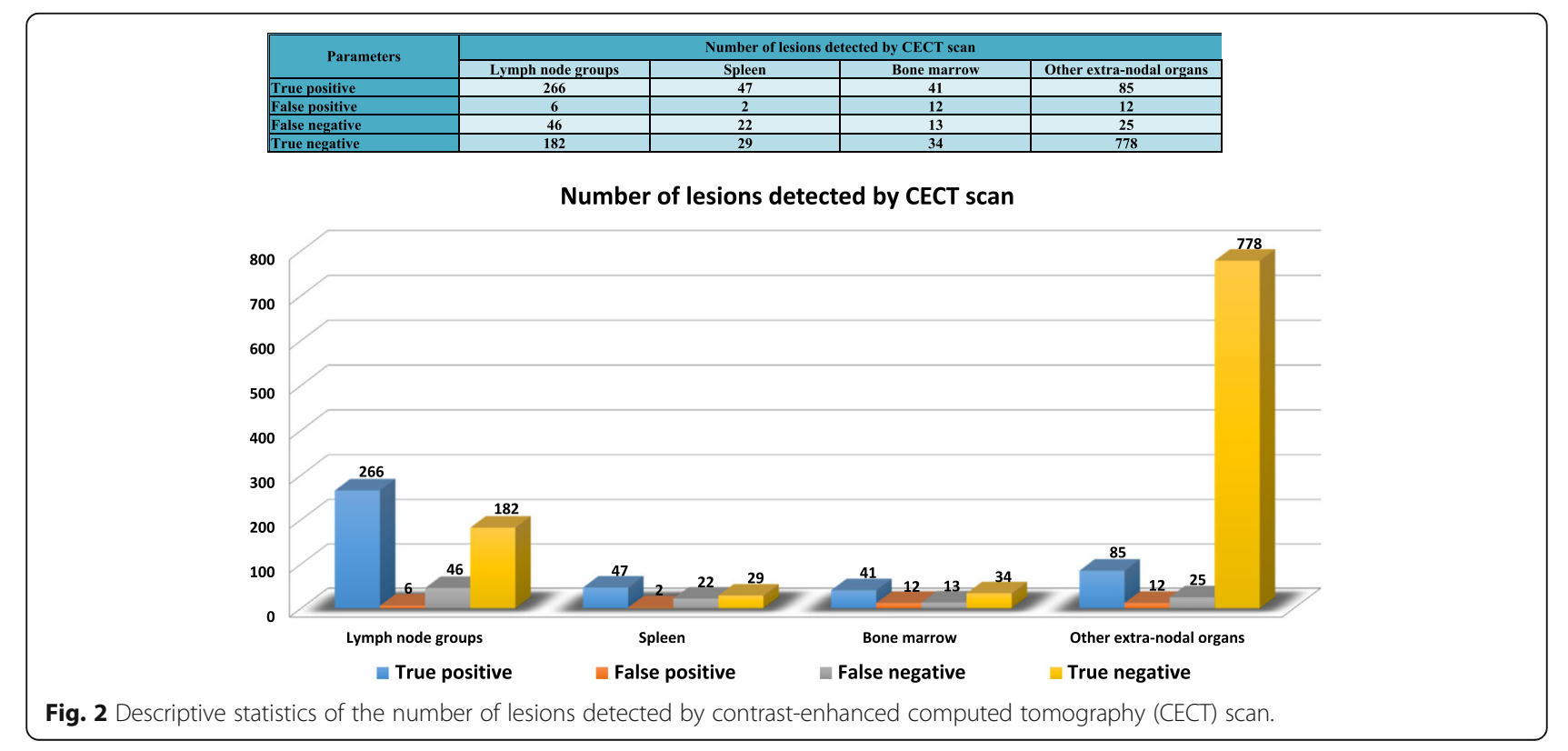


Table 3 Descriptive statistics of the number of lesions detected by PET/CT scan

\begin{tabular}{|c|c|c|c|c|c|}
\hline \multirow[t]{2}{*}{ Parameters } & \multicolumn{5}{|c|}{ Number of lesions detected by PET/CT scan } \\
\hline & Lymph Node groups & Spleen & Bone marrow & Other extra-nodal organs & Total \\
\hline True positive & 312 & 79 & 54 & 100 & 545 \\
\hline False positive & 4 & 1 & 1 & 4 & 10 \\
\hline False negative & 3 & 0 & 0 & 0 & 3 \\
\hline True negative & 181 & 20 & 45 & 796 & 1042 \\
\hline Total examined sites in 100 patients & 500 & 100 & 100 & 900 & 1600 \\
\hline
\end{tabular}

- For lymph nodal involvement: PET/CT sensitivity $97.5 \%$, specificity $94 \%$, and accuracy 98\% were higher than CECT sensitivity $83.1 \%$, specificity $94 \%$, and accuracy $89,6 \%$ respectively with significant difference $(P>0.005)$ (Table 6; Fig. 6).

- For splenic involvement: PET/CT sensitivity 95.2\%, specificity $98 \%$, and accuracy $99 \%$ were higher than CECT sensitivity $87.6 \%$, specificity $86.6 \%$, and accuracy $76 \%$.

- For bone marrow involvement: PET/CT sensitivity 93.7\%, specificity $96 \%$, and accuracy $99 \%$ were higher than CECT sensitivity $88.6 \%$, specificity $86.2 \%$, and accuracy $75 \%$.

- For other extranodal organs involvement: PET/CT sensitivity $94 \%$, specificity $96.2 \%$, and accuracy $99.5 \%$ were higher than CECT sensitivity $80 \%$, specificity $88.6 \%$, and accuracy $95.9 \%$.

\section{Regarding lymphoma staging according to Lugano classification by CECT and PET/CT}

Discordant staging by CECT and PET/CT was found in $23 \%$ of the patients. Lymphoma was upstaged by $\mathrm{PET} / \mathrm{CT}$ in $17 \%$ of patients, with major changes in $12 \%$; i.e., upstaging from stages I or II to stages III or IV; and was downstaged in $6 \%$ of the patients as follows :CECT diagnosed $8 \%$ of the patients as stage I; 3 of them were diagnosed by PET/CT as stage IV which means upstaging with major changes of $3 \%$ patients by PET/CT.CECT diagnosed $27 \%$ of the patients as stage II; 4 of them were diagnosed by PET/ CT as stage III and other 5 of them were diagnosed by PET/CT as stage IV which means upstaging with major changes of $9 \%$ patients by PET/CT. CECT diagnosed $28 \%$ of the patients as stage III; 5 of them were diagnosed by PET/CT as stage IV which means upstaging of $5 \%$ patients by PET/CT. CECT

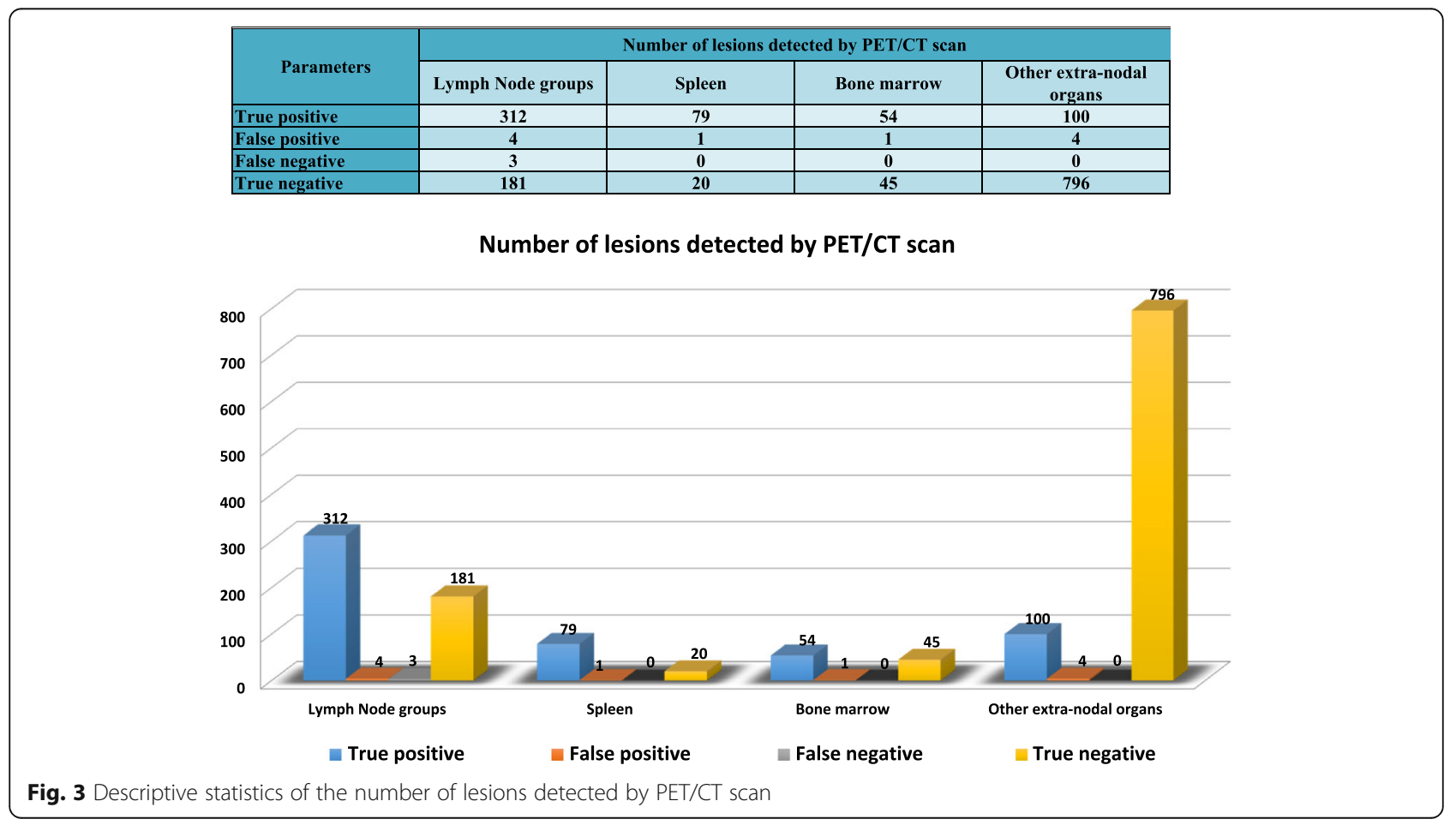


Table 4 Agreement between CECT and PET/CT in detection of involved groups of lymph nodes

\begin{tabular}{|c|c|c|c|c|c|c|c|}
\hline \multirow[t]{3}{*}{ Lymph node groups } & & \multicolumn{4}{|c|}{ Involvement of lymph node groups by: } & \multirow[t]{3}{*}{$\mathrm{X} 2$} & \multirow[t]{3}{*}{$P$ value } \\
\hline & & \multicolumn{2}{|c|}{ CECT } & \multicolumn{2}{|c|}{$\mathrm{PET} / \mathrm{CT}$} & & \\
\hline & & No & $\%$ & No & $\%$ & & \\
\hline \multirow[t]{2}{*}{ Cervical } & Yes & 48 & $48.0 \%$ & 70 & $70.0 \%$ & 10.004 & 0.002 \\
\hline & No & 52 & $52.0 \%$ & 30 & $30.0 \%$ & & \\
\hline \multirow[t]{2}{*}{ Axillary } & Yes & 44 & $44.0 \%$ & 51 & $51 \%$ & 0.845 & 0.396 \\
\hline & No & 56 & $56.0 \%$ & 49 & $49 . \%$ & & \\
\hline \multirow[t]{2}{*}{ Mediastinal and hilar } & Yes & 66 & $66.0 \%$ & 73 & $73.0 \%$ & 1.156 & 0.282 \\
\hline & No & 34 & $34.0 \%$ & 27 & $27.0 \%$ & & \\
\hline \multirow[t]{2}{*}{ Abdomino-pelvic } & Yes & 67 & $67.0 \%$ & 74 & $74.0 \%$ & 1.178 & 0.352 \\
\hline & No & 33 & $33.0 \%$ & 26 & $26.0 \%$ & & \\
\hline \multirow[t]{2}{*}{ Inguinal } & Yes & 40 & $40.0 \%$ & 45 & $45.0 \%$ & 0.512 & 0.567 \\
\hline & No & 60 & $60.0 \%$ & 55 & $55.0 \%$ & & \\
\hline
\end{tabular}

diagnosed $37 \%$ of the patients as stage IV; 6 of them were diagnosed by PET/CT as stage III which means downstaging of $6 \%$ patients by PET/CT (Table 7; Fig. 7).

\section{Discussion}

Many lymphomas are potentially curable with their prognosis depends on the stage and histological type [5]. CT scan used to be the cornerstone of imaging in lymphoma and was playing a crucial role in staging. Currently, the advances in molecular imaging with18F-FDG PET/CT scan have facilitated the diagnosis, staging, and response assessment in lymphoma patients [3]. Integrated $\mathrm{PET} / \mathrm{CT}$ offers the advantage of combining functional and anatomical information and better attenuation correction

\begin{tabular}{|c|c|c|c|}
\hline \multirow{2}{*}{\multicolumn{2}{|c|}{ Lymph node groups }} & \multicolumn{2}{|c|}{$\begin{array}{l}\text { Percent of involved groups of lymphnodes by } \\
\text { CECT and PET/CT }\end{array}$} \\
\hline & & CECT & PET/CT \\
\hline \multirow{2}{*}{ Cervical } & Yes & $48.00 \%$ & $70.00 \%$ \\
\hline & No & $52.00 \%$ & $30.00 \%$ \\
\hline \multirow{2}{*}{ Axillary } & Yes & $44.00 \%$ & $51 \%$ \\
\hline & No & $56.00 \%$ & $49.00 \%$ \\
\hline \multirow{2}{*}{$\begin{array}{l}\text { Mediastinal \& } \\
\text { hilar }\end{array}$} & Yes & $66.00 \%$ & $73.00 \%$ \\
\hline & No & $34.00 \%$ & $27.00 \%$ \\
\hline \multirow{2}{*}{$\begin{array}{l}\text { Abdomino- } \\
\text { pelvic } \\
\end{array}$} & Yes & $67.00 \%$ & $74.00 \%$ \\
\hline & No & $33.00 \%$ & $26.00 \%$ \\
\hline \multirow{2}{*}{ Inguinal } & Yes & $40.00 \%$ & $45.00 \%$ \\
\hline & No & $60.00 \%$ & $55.00 \%$ \\
\hline
\end{tabular}

Percent of involved groups of lymphnodes by CECT and PET/CT

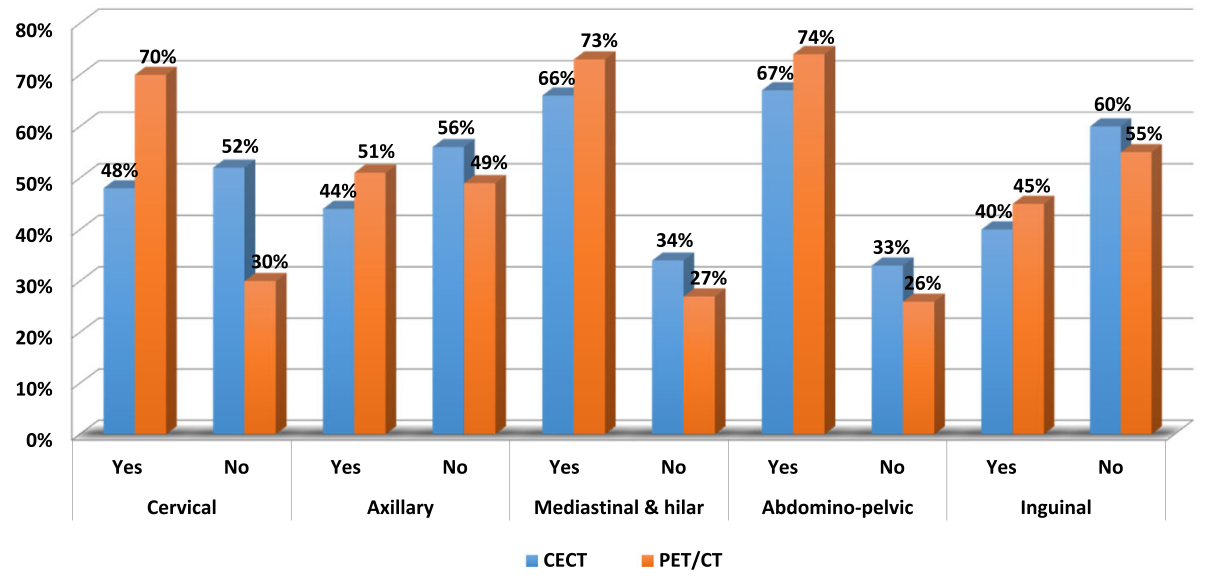

Fig. 4 Agreement between CECT and PET/CT in detection of involved groups of lymph nodes 
Table 5 Descriptive statistics of the involved other extra nodal organs by PET/CT and their SUVmax

\begin{tabular}{llll}
\hline Involved extra nodal organs & & SUV max & Percent of involvement by PET/CT \\
\hline High FDG uptake & Lung & 44.0 & $19 \%$ \\
& GIT (Stomach) & 33.4 & $10 \%$ \\
& Head and Neck & 32.7 (submandibular gland) & $12 \%$ \\
Moderate FDG uptake & Reno-Adrenal & 30.8 (Kidney) & $12 \%$ \\
& Skin and subcutaneous & 27.3 & $9 \%$ \\
Low FDG uptake & Breast & 25.0 & $4 \%$ \\
& Muscle & 21.1 & $3 \%$ \\
& Liver & 7 & $14 \%$ \\
& GIT & 6.5 (peritoneum) & $6 \%$ \\
& Chest & 5.5 (pleura) & $7 \%$ \\
\hline
\end{tabular}

[4]. The purpose of this study is to evaluate and compare the diagnostic performance of18F-FDG$\mathrm{PET} / \mathrm{CT}$ and CECT in the initial assessment of nodal and extra-nodal lymphomatous lesions. For this purpose, 100 patients diagnosed as lymphoma, which proved and classified by histopathology, were initially assessed and staged by CECT and 18F-FDG PET/ CT (Figs. 8, 9, 10, 11, 12, 13, 14, 15, 16, and 17).

\section{Regarding demographic data}

In this study, the percent of patients having NHL $66 \%$ was higher than those having HL 34\%; this was agreed with Raanani P et al. [15] whose study stated that the incidence of NHL $68 \%$ was higher than that of HL $35 \%$. Also, Mozaffer R and Sadiqa S's [16] study stated that $81.6 \%$ of their patients had NHL compared to $18.4 \%$ had HL. The same lymphoma type predilection was resulted by Roman E and Smith A [17]., while the study of Ricard F et al. [18] was carried out on $50.8 \%$ of cases with NHL and $49.2 \%$ with HL. In our study, NHL patients showed higher percent of males $39 \%$ than females $27 \%$, also HL patients showed higher percent of males $22 \%$ than females $12 \%$ which emphasizes that male affection by both types of lymphoma is higher than female affection. The same sex predilection was resulted by Roman E and Smith A [17]. In our study, the higher percent $27 \%$ of cases was at the age group $50-<60$ years distributed as $24 \%$ had NHL and 3\% had HL, while the lower percent $16 \%$ was at the age group of $20-<30$ years distributed as $12 \%$ had HL and $4 \%$ had NHL. This was agreed with Rodriguez B et al. [19] who stated that mean age of NHL $52 \pm 15$ while for HL the mean age was $31 \pm 12$.

\section{Regarding total lesions}

In our study, PET/CT diagnosed a total number of 545 involved regions with sensitivity $96.6 \%$, specificity $98.8 \%$, and accuracy 99\% which was higher than those diagnosed by CECT; 439 involved regions with sensitivity
$87.5 \%$, specificity $85.7 \%$, and accuracy $88 \%$; la Fougere C et al.'s [20] study showed that PET/CT sensitivity $97 \%$ was higher than that of CECT $87.5 \%$, also PET/CT specificity $99 \%$ was higher than that of CECT $85.5 \%$ as follows.

\section{Regarding lymph nodal involvement}

Our study resulted that PET/CT detected 312 true positive nodal group involvement with sensitivity $97.5 \%$, specificity $94 \%$, and accuracy $98 \%$ which was higher than CECT that detected 266 true positive nodal group involvement with sensitivity $83.1 \%$, specificity $94 \%$, and accuracy $89.6 \%$, denoting significant difference $(P>0.005)$. Also, there were 46 false negative lymph node groups on CECT that decreased to only 3 groups by PET/CT. This was in agreement with Ricard F et al. [18] who stated that the sensitivity of PET/CT 99\% was higher than the sensitivity of CECT $85 \%$ and also detected in their study 32 false negative lymph node groups by CECT that was corrected to 3 groups by PET/CT. Kwee T et al. [21] mentioned that some subtypes of lymphoma, namely small cell lymphocytic lymphoma/chronic lymphocytic leukemia may manifest as an increased number of small LNs, which declares that the size criteria used for the morphological assessment of lymph nodes in oncohematology is insufficient and the metabolic status of lymph nodes by PET scan should be integrated even if their size seems to be normal on CT. The most common involved lymph node group, in this study, was the abdomino-pelvic group, while the least common was inguinal group. In agreement with our study, the most common affected LN group in Mozaffer $\mathrm{R}$ and Sadiqa S's [16] study was the abdominopelvic group and the least common was the inguinal group, while the most common affected LN group in Rodriguez B et al.'s [19] study was the mediastinal group and the least common was the abdominal group. 


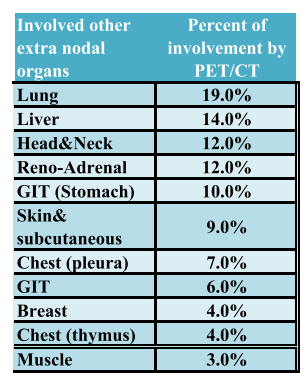

(A) Percent of Involved other extra nodal organs by PET/CT
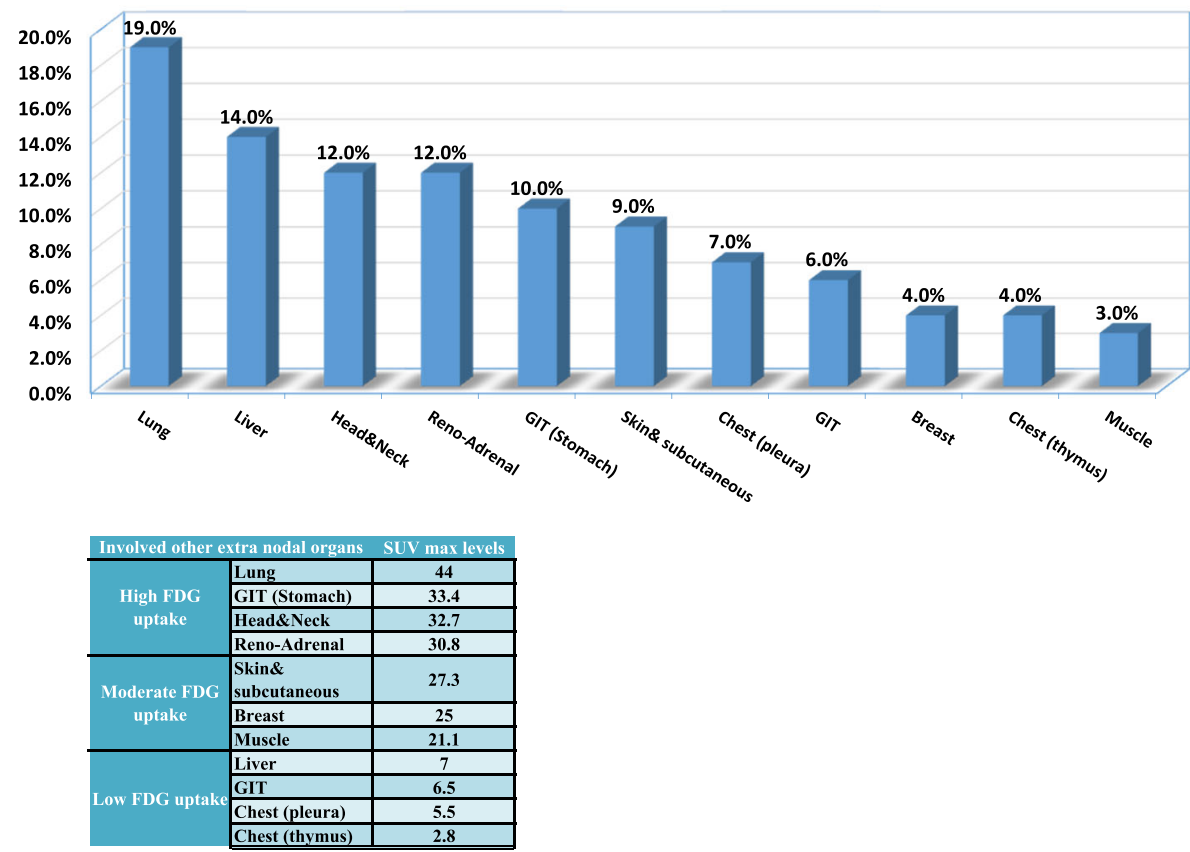

(B) SUV max levels of Involved other extra nodal organs by PET/CT

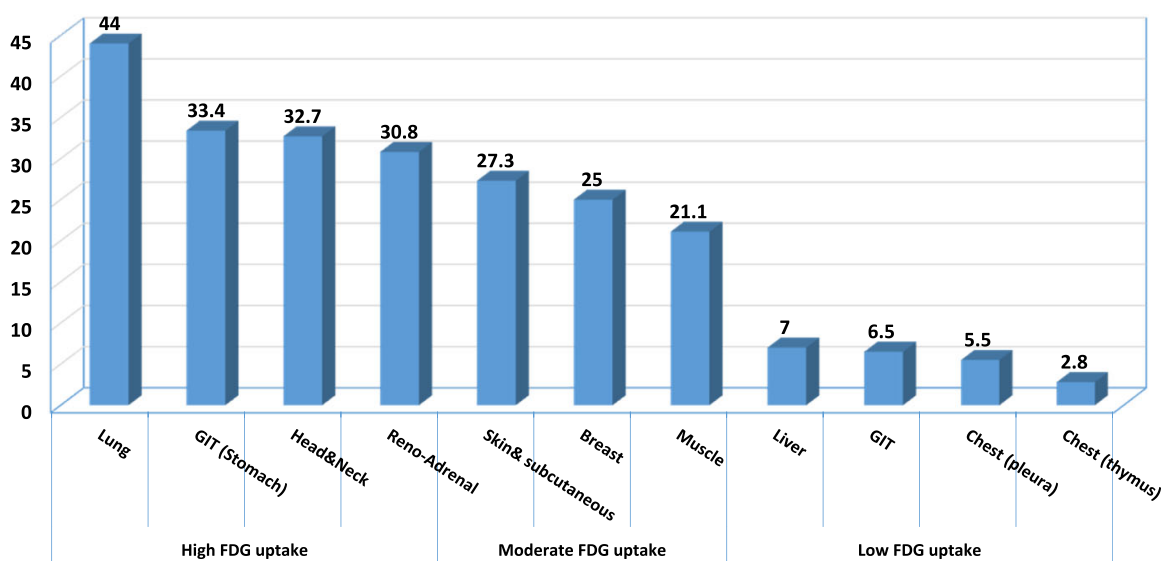

Fig. 5 a Descriptive statistics of the involved other extra nodal organs by PET/CT. b SUVmax of involved other extra nodal organs

\section{Regarding splenic involvement}

In this study, splenic involvement was truly positive in $79 \%$ of the patients by PET/CT with sensitivity $95.2 \%$, specificity 98\%, and accuracy 99\% which was higher than CECT that detected true positive lesions in $47 \%$ of the patients with sensitivity $87.6 \%$, specificity $86.6 \%$, and accuracy $76 \%$. There were $22 \%$ of the patients with false negative results when assessed by CECT alone that was corrected to zero\% by 
Table 6 Diagnostic performance of CECT and PET/CT scans

\begin{tabular}{|c|c|c|c|c|c|c|}
\hline \multirow[t]{2}{*}{ Parameters } & \multicolumn{3}{|l|}{ CECT } & \multicolumn{3}{|l|}{$\mathrm{PET} / \mathrm{CT}$} \\
\hline & Sensitivity & Specificity & accuracy & Sensitivity & Specificity & Accuracy \\
\hline Lymph nodes & $83.1 \%$ & $94 \%$ & $89.6 \%$ & $97.5 \%$ & $94 \%$ & $98 \%$ \\
\hline Spleen & $87.6 \%$ & $86.6 \%$ & $76 \%$ & $95.2 \%$ & $98 \%$ & $99 \%$ \\
\hline Bone marrow & $88.6 \%$ & $86.2 \%$ & $75 \%$ & $93.7 \%$ & $96 \%$ & $99 \%$ \\
\hline Extra-nodal & $80 \%$ & $88.6 \%$ & $95.9 \%$ & $94 \%$ & $96.2 \%$ & $99.5 \%$ \\
\hline Total mean & $87.5 \%$ & $85.7 \%$ & $88 \%$ & $96.6 \%$ & $98.8 \%$ & $99 \%$ \\
\hline
\end{tabular}

PET/CT. Close results were concluded by De Jong $\mathrm{P}$ et al. [22] who stated that PET/CT sensitivity and specificity in detecting splenic involvement were 100\% and 95\% versus 91\% and 96\% for CECT and confirmed the importance of metabolic status shown on PET/CT to discover splenic lesions with density similar to splenic tissue that could not be differentiated by CECT alone.

\section{Regarding bone marrow involvement}

In our study, bone marrow involvement was truly positive in $54 \%$ of the patients by PET/CT with sensitivity 93.7\%, specificity of 96\%, and accuracy $99 \%$ which was higher than CECT that detected true positive lesions in $41 \%$ of the patients with sensitivity $88.6 \%$, specificity $86.2 \%$, and accuracy $75 \%$. There were $13 \%$ of the patients with false negative bone marrow involvement when assessed by CECT alone that was corrected to zero\% by PET/CT. Also, the axial skeleton was the most commonly involved. In agreement with our results, Kwee $\mathrm{T}$ et al. [21] who mentioned that PET/CT is more sensitive 95\% than
CECT 86\%. Othman A et al. [23] detected that bone marrow lymphoma mainly affecting the axial skeleton more than the appendicular skeleton.

\section{Regarding other extranodal organ involvement}

In our study, PET/CT detected 100 true positive extranodal organ involvements with sensitivity $94 \%$, specificity $96.2 \%$, and accuracy $99.5 \%$ which was higher than CECT that detected 85 true positive extranodal organ involvements with sensitivity $80 \%$, specificity $88.6 \%$, and accuracy $95.9 \%$. There were 25 false negative extranodal organ involvements when assessed by CECT alone that was corrected to zero\% by PET/CT. In agreement with our results, Ricard F et al. [18] stated that PET/CT sensitivity in detection of extranodal lymphomatous involvement $88 \%$ was higher than that of CECT alone $78 \%$ and that 9 false negative extranodal results by CECT were corrected to 5 by PET/CT. The most common involved extranodal organs in our study were lung 19\%, GIT (mainly the stomach) 16\%, liver $14 \%$ and renoadrenal $12 \%$. In Das J et al. [22], the most frequently involved

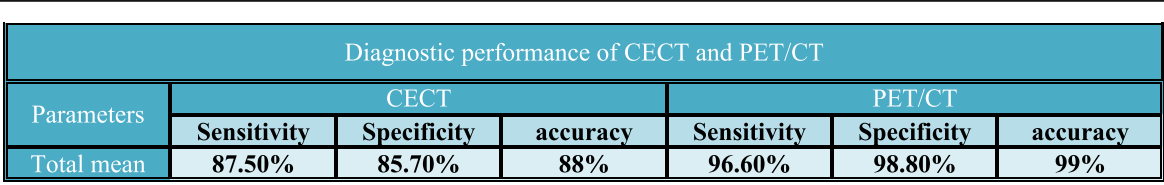

Diagnostic performance of CECT and PET/CT

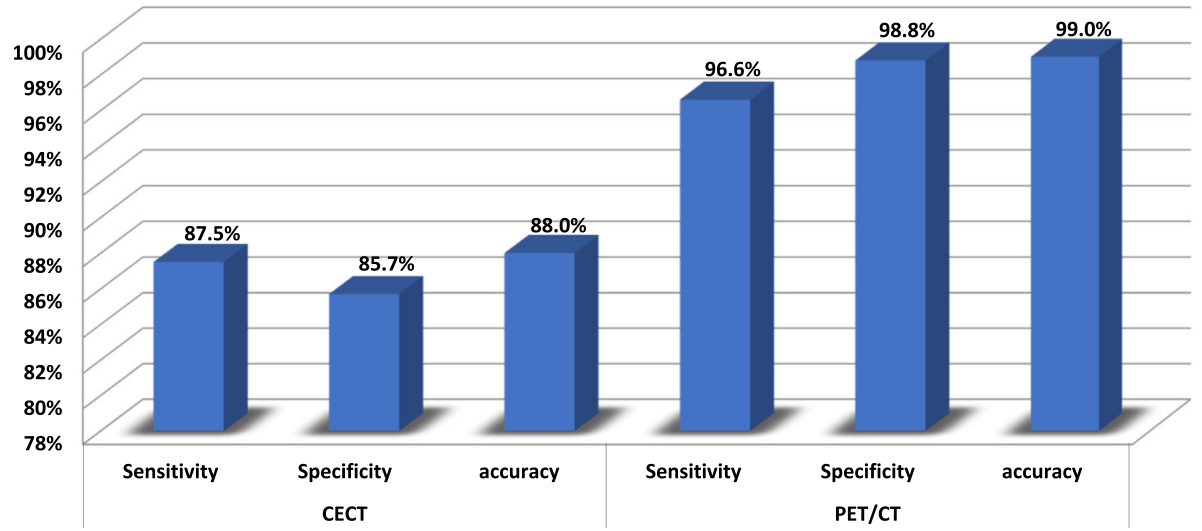

Fig. 6 Diagnostic performance of CECT and PET/CT scans 
Table 7 Changes in staging according to Lugano classification between CECT and PET/CT

\begin{tabular}{llllll}
\hline Lugano stage & \multicolumn{4}{l}{ PET/CT scan } \\
\cline { 2 - 6 } CECT scan & I & II & III & IV & Total \\
\hline I & 5 & - & - & 3 & 8 \\
II & - & 18 & 4 & 5 & 27 \\
III & - & - & 23 & 5 & 28 \\
IV & - & - & 6 & 31 & 37 \\
Total & 5 & 18 & 33 & 44 & 100 \\
\hline
\end{tabular}

extranodal organs were GIT $14.8 \%$ (stomach is commonest site), followed by head and neck region $10 \%$ (including tonsils, pharynx, tongue, and orbit), lung $8 \%$, and liver 5\%, while Othman A et al.'s [23] study stated that the most common involved extranodal organs were head and neck $18 \%$ and GIT $15 \%$. In this study, the organs showing the highest SUV max by PET/CT were lung 44.0, GIT (stomach) 33.4, head and neck (submandibular glands) 32.7, and renoadrenal (kidney) 30.8, while the organs showing the lowest SUV max were chest (thymus 2.80), chest (pleura 5.50), GIT (peritoneum 6.50), and liver 7.00. Othman A et al.'s [23] study resulted that the organs showing the highest SUV max were kidney 73.0, oropharynx 60.0 , tonsils 40 , and thyroid 37.0, while organs showing the lowest SUV max were pancreas 6.0, peritoneum 8.2, and suprarenal 9.0.

\section{Regarding lymphoma staging according to Lugano classification}

Differences in staging by PET/CT and CECT were found in our study. Discordant staging by both modalities was found in $23 \%$ of the patients. Lymphoma was upstaged by PET/CT in $17 \%$ of patients; with major changes in $12 \%$ (i.e., upstaging from stages I or II to stages III or IV) and downstaged in 6\%. In agreement with our results, Ricard F et al. [18] who stated changes in the staging of $20 \%$ of the patients with upstaging in $17 \%$ and downstaging in 3\% with major changes in 10\%. Also, Othman A et al.'s [23] study mentioned that $10 \%$ of the patients were upstaged while $5 \%$ were downstaged after PET/CT. Luminari et al. [24] resulted that PET/CT helped in upstaging of $11 \%$ and downstaging of $1 \%$ of patients, while Raanani P et al.'s [15] study revealed changes in staging of $39 \%$ of the patients after PET/CT with $33 \%$ upstaging and $6 \%$ downstaging. This change in staging was explained by the higher ability of PET/CT over CECT in detection of involved sites either with normal morphology; as normal-sized lymph nodes or hidden and missed by its isodensity in extra nodal sites, by detection of increased activity in the form of high FDG uptake.

\section{Conclusion}

FDG-PET/CT scan should replace CECT in the initial assessment and staging of lymphoma, as FDG$\mathrm{PET} / \mathrm{CT}$ scan showed higher sensitivity, specificity, and accuracy which led to alteration of disease staging with marked effects on the decision of treatment regimens.

\section{Limitation of this study}

For clear practical and ethical reasons, it was not possible to take biopsies from all nodal and extranodal suspected sites in order to determine a gold

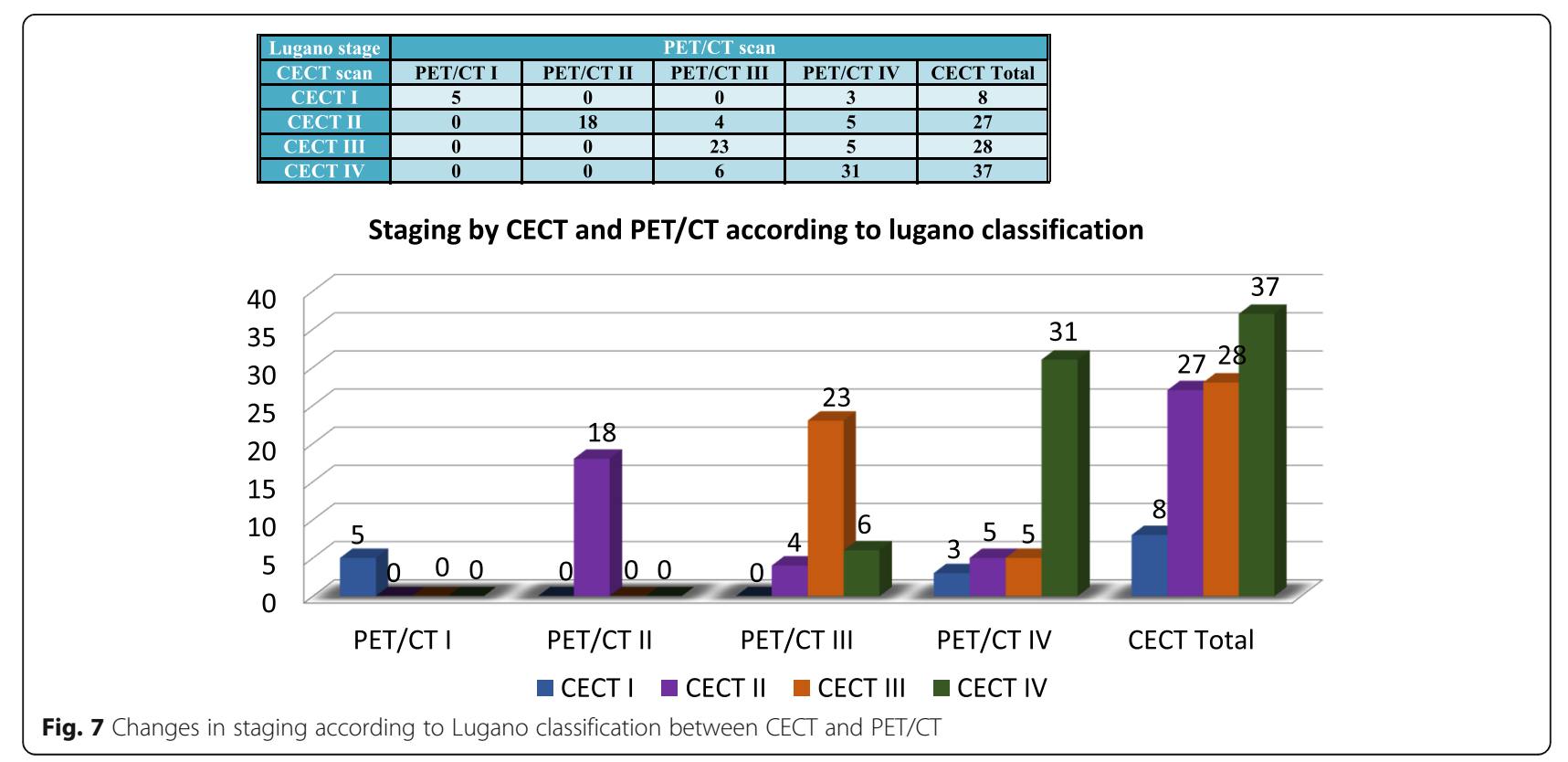



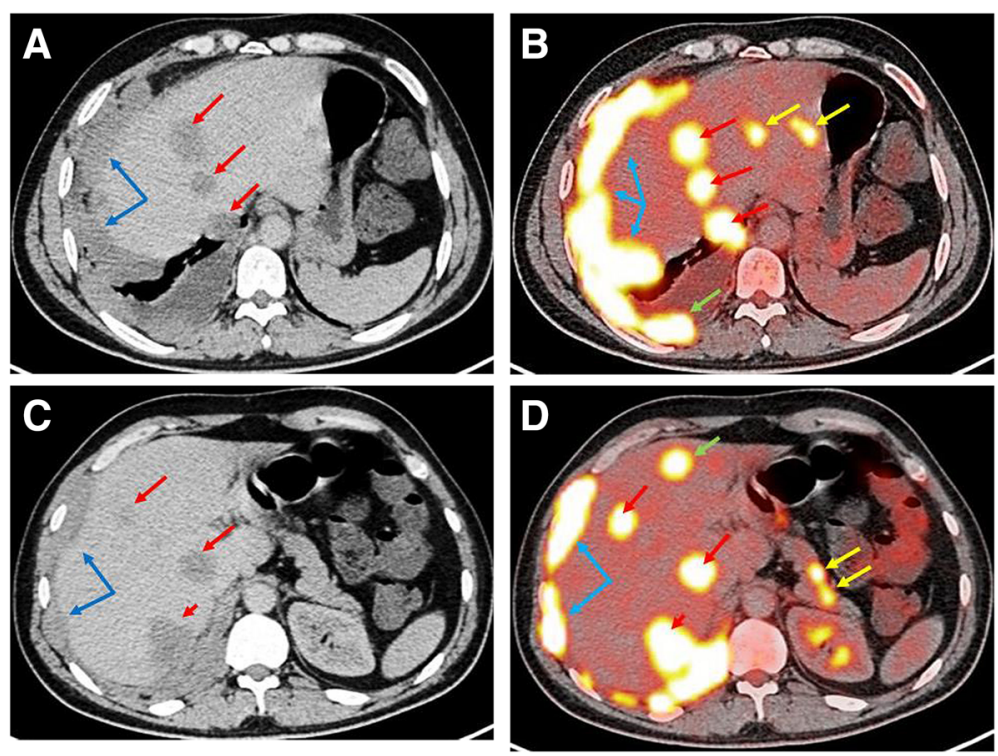

Fig. 8 Male patient 63 years old diagnosed and proven by histopathology as hepatic non-Hodgkin lymphoma. a Axial contrast-enhanced CT (CECT) of the abdomen showing three hypodense non enhancing hepatic focal lesions seen at segment VIII (red arrows) and peri hepatic peritoneal nodular thickening (blue arrows). b Axial PET/CT showing avid FDG uptake of the three hepatic focal lesions seen at segment VIII (red arrows) and peri hepatic peritoneal nodular thickening (blue arrows) previously noted on CECT, with another FDG avid two hepatic focal lesions seen at segment IV (yellow arrows) and right pleural nodular thickening underlying the pleural effusion (green arrow), not seen on CECT. c Axial CECT showing three hypo dense non enhancing hepatic focal lesions; two of them seen at segment VIII (red long arrows) and one seen at segment VII (red short arrow) with peri hepatic peritoneal nodular thickening (blue arrows). d Axial PET/CT showing avid FDG uptake of the three hepatic focal lesions seen at segment VIII (red long arrows) and at segment VII (red short arrow) with peri hepatic peritoneal nodular thickening (blue arrows) previously noted on CECT, with another FDG avid one hepatic focal lesion seen at segment IV (green arrow) and pancreatic two lymphomatous focal lesions (yellow arrows), not seen on CECT
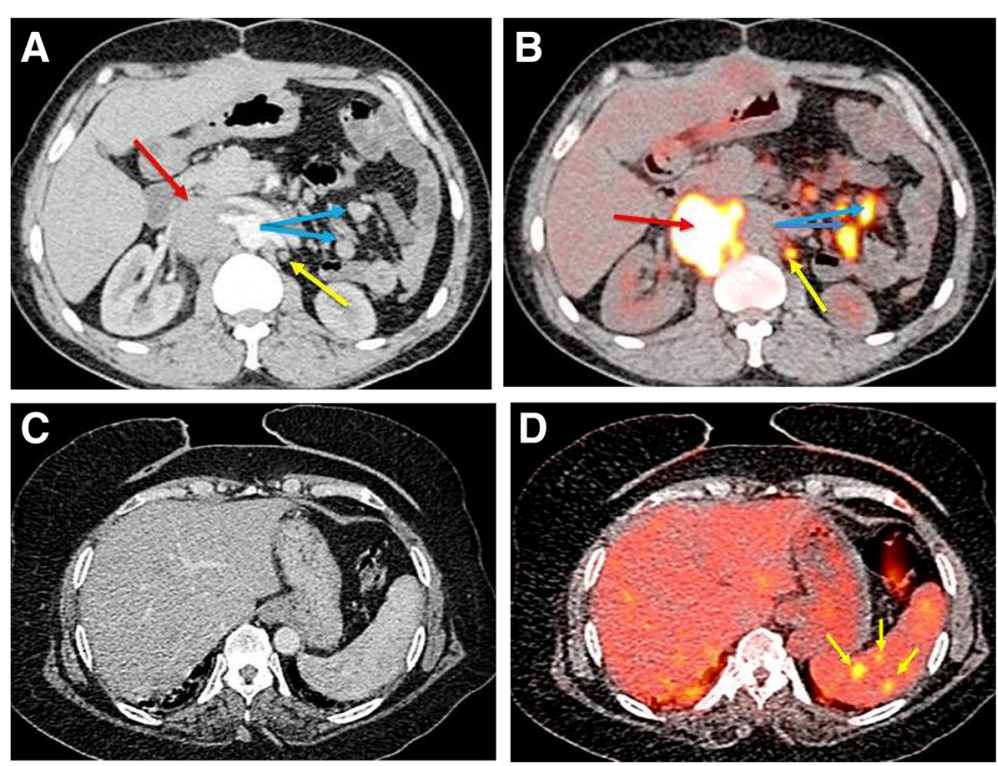

Fig. 9 Female patient 44 years old, diagnosed and proven by histopathology as abdominal lymph nodal Hodgkin lymphoma. a Axial contrast enhanced CT (CECT) of the abdomen showing amalgamated aortocaval lymph nodes (red arrow) and enlarged mesenteric lymph nodes (blue arrows), with a small left paraaortic lymph node $8 \mathrm{~mm}$ in its short axis (yellow arrow). b Axial PET/CT showing avid FDG uptake of the amalgamated aortocaval lymph nodes (red arrow) and enlarged mesenteric lymph nodes (blue arrows) previously seen on CECT, with avid FDG uptake of the small left paraaortic lymph node (yellow arrow). c Axial CECT showing no splenic focal lesions. d Axial PET/CT showing increased FDG uptake of three splenic focal lesions (yellow arrows) not seen on CECT 

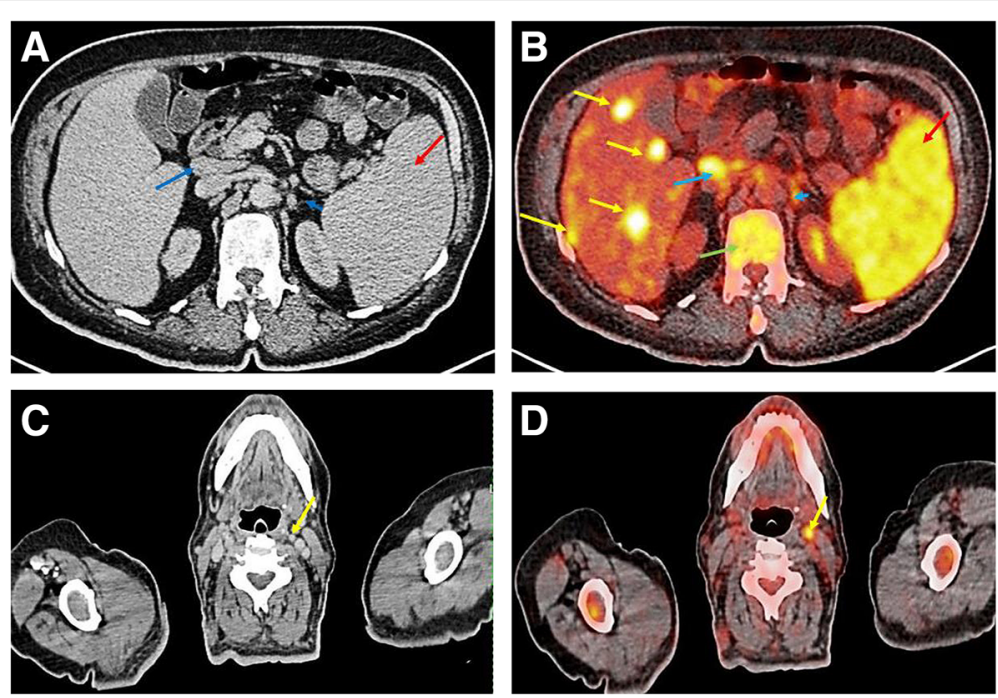

Fig. 10 Male patient 51 years old diagnosed and proven by histopathology as splenic non-Hodgkin lymphoma. a Axial contrast enhanced CT (CECT) of the abdomen showing splenomegaly (red arrow) and enlarged portahepatis lymph node (blue long arrow), with small left paraaortic lymph node $9 \mathrm{~mm}$ (blue short arrow). $\mathbf{b}$ Axial PET/CT showing diffuse avid FDG uptake of the enlarged spleen (red arrow), the enlarged portahepatis lymph node (blue long arrow) and small left paraaortic lymph node (blue short arrow) previously noted on CECT, with avid FDG uptake of four hepatic focal lesions (yellow arrows) and bone marrow of the vertebral body (green arrow) not seen on CECT. c Axial CECT showing small $7 \mathrm{~mm}$ cervical lymph node (yellow arrow). $\mathbf{d}$ Axial PET/CT showing increased FDG uptake of the small cervical lymph node (yellow arrow)

standard. So, the standard reference was based on clinical data and other monitoring factors including clinical history; physical examination; laboratory work-up such as cell blood count, serum creatinine, urea, liver function tests, lactate dehydrogenase, b2 microglobulin, and viral serologies; imaging findings such as ultrasound and MRI when necessary; and iliac crest bone marrow biopsy. This limitation is also inherent in other previously published studies such as Raanani P et al. [15].
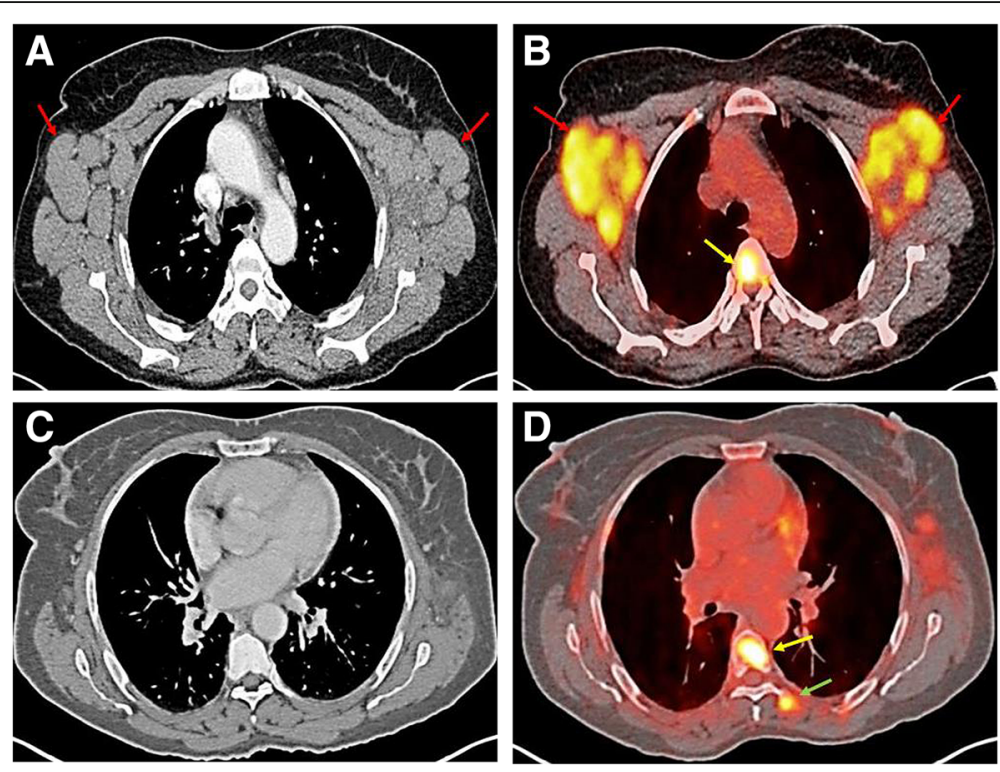

Fig. 11 Female patient 52 years old diagnosed and proven by histopathology as axillary lymph nodal Hodgkin lymphoma. a Axial contrast enhanced CT (CECT) showing bilateral enlarged axillary lymph nodes (red arrows). b Axial PET/CT showing avid FDG uptake of the bilateral enlarged axillary lymph nodes (red arrows) previously seen on CECT, with increased FDG uptake of the bone marrow lesion of the dorsal spine (yellow arrow) not seen on CECT. c Axial CECT showing no bone marrow or soft tissue lesions. d Axial PET/CT showing avid FDG uptake of bone marrow lesion of the dorsal vertebra (yellow arrow) and left paravertebral soft tissue focal lesion (green arrow) not seen on CECT 

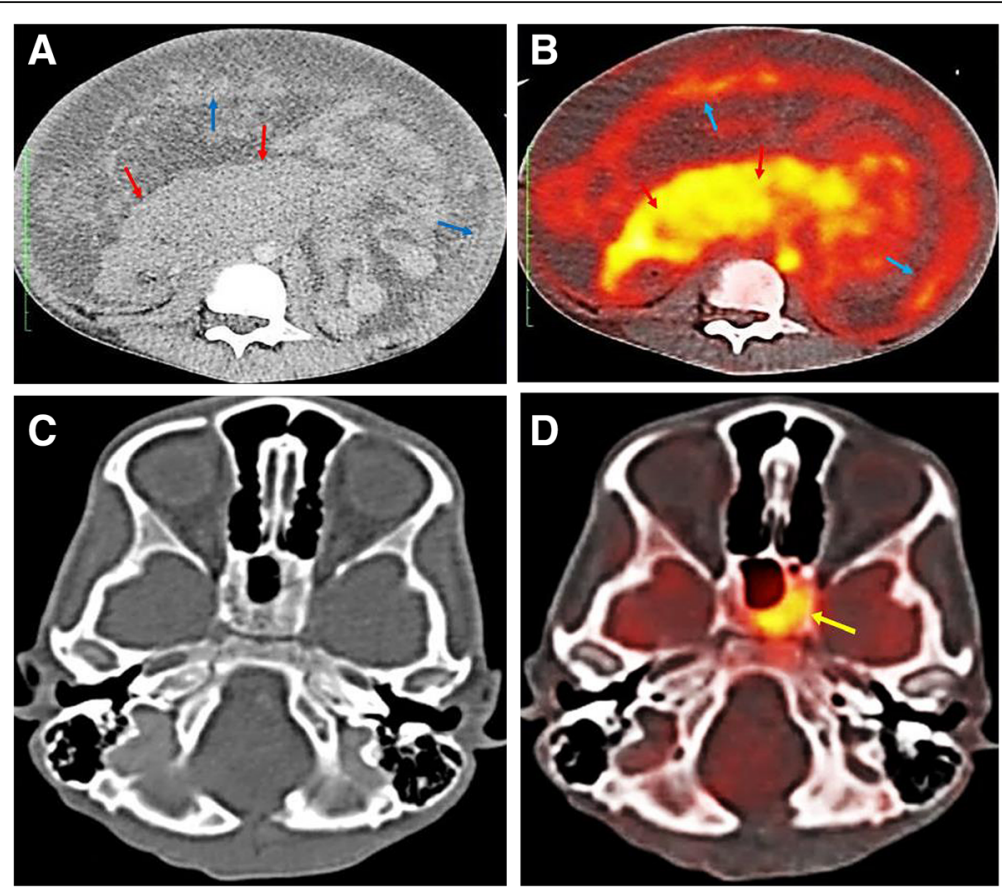

Fig. 12 Male patient 66 years old diagnosed and proven by histopathology as abdominal lymph nodal Non- Hodgkin lymphoma. a Axial contrast enhanced CT (CECT) showing sizable amalgamated paraaortic and mesenteric lymph nodes (red arrows) and peritoneal thickening (blue arrows). b Axial PET/CT showing avid FDG uptake of the amalgamated paraaortic and mesenteric lymph nodes (red arrows) and peritoneal thickening (blue arrows) previously seen on CECT. c Axial CT of skull base (bone window) showing no lesion could be detected. d Axial PET/CT showing avid FDG uptake of lymphomatous focal bone marrow lesion of the left side of the sphenoid bone (yellow arrow) not seen on CECT
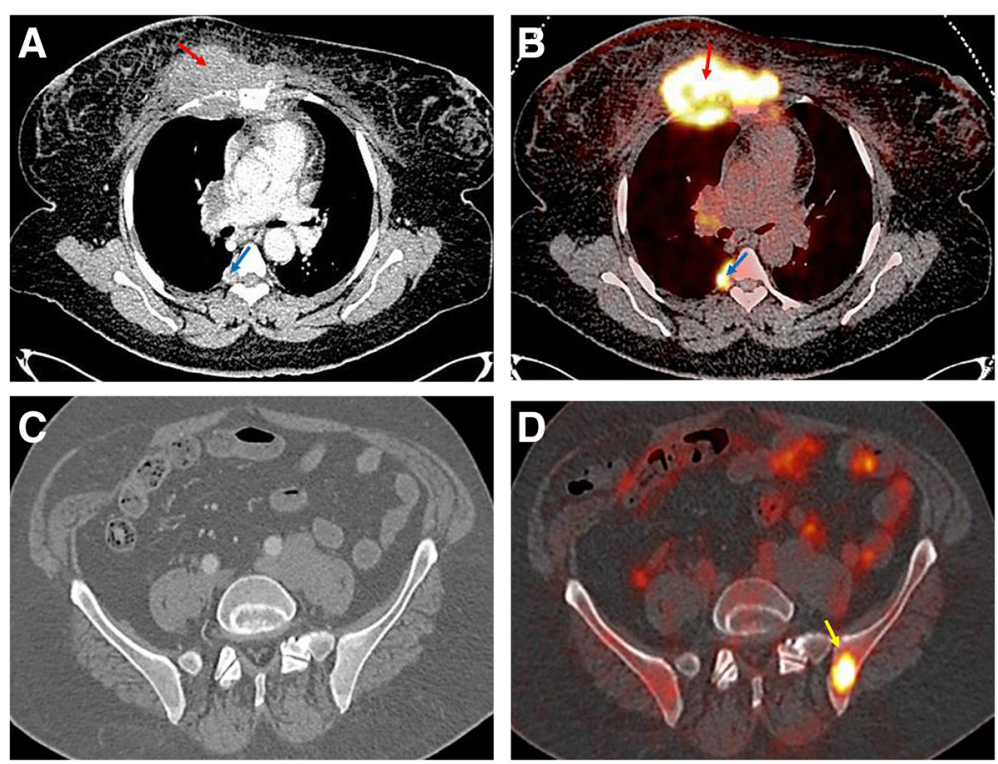

Fig. 13 Female patient 47 years old diagnosed as having anterior chest wall mass lesion, proven by histopathology as non-Hodgkin lymphoma. a Axial contrast enhanced CT (CECT) showing sizable soft tissue mass lesion of the right anterior chest wall infiltrating the underlying bones (red arrow), with small focal right paravertebral pleural thickening (blue arrow). b Axial PET/CT showing avid FDG uptake of the anterior chest wall lesion (red arrow) and pleural thickening (blue arrow) previously seen on CECT. c Axial CECT of the pelvis ,bone window, showing no pelvic osseous lesion. $\mathbf{d}$ Axial PET/CT showing avid FDG uptake of focal bone marrow lesion of the left side of the hip bone (yellow arrow) not seen on CECT 

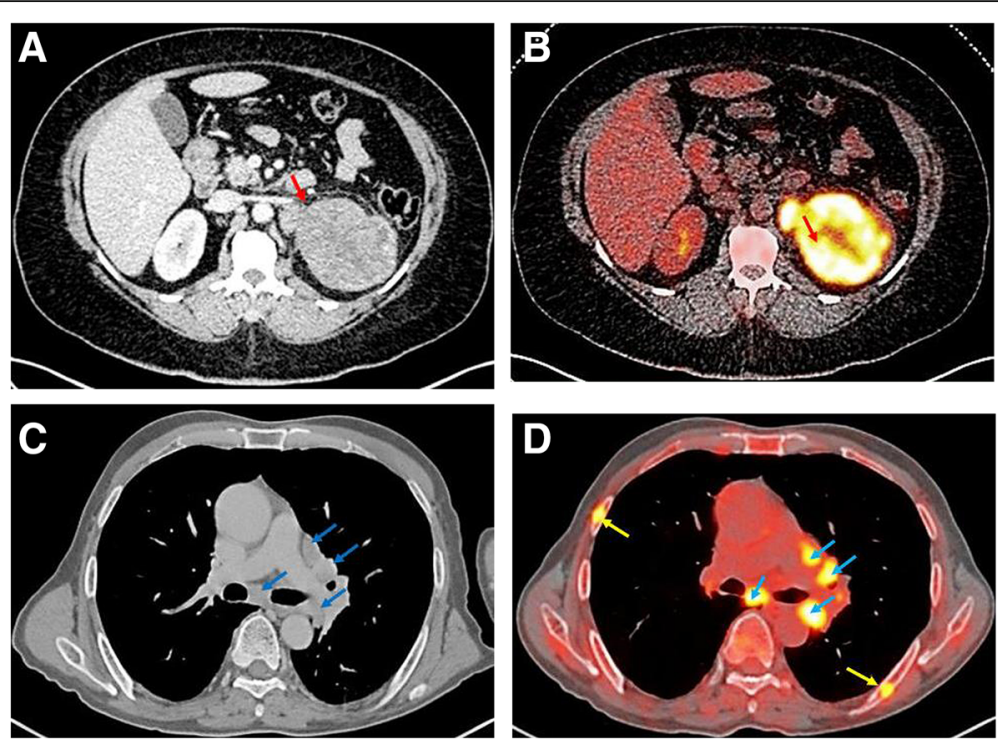

Fig. 14 Male patient 65 years old diagnosed and proven by histopathology as renal non-Hodgkin lymphoma. a Axial contrast enhanced CT (CECT) of the abdomen showing diffusely enlarged hypo dense non enhancing left kidney (red arrow). b Axial PET/CT showing avid FDG uptake of the infiltrated left kidney (red arrow) previously seen on CECT. c Axial CECT of the chest showing enlarged mediastinal lymph nodes (blue arrows) with no osseous lesion could be detected. $\mathbf{d}$ Axial PET/CT showing avid FDG uptake of the enlarged mediastinal lymph nodes (blue arrows), with bilateral costal focal bone marrow lesions (yellow arrows) not seen on CECT
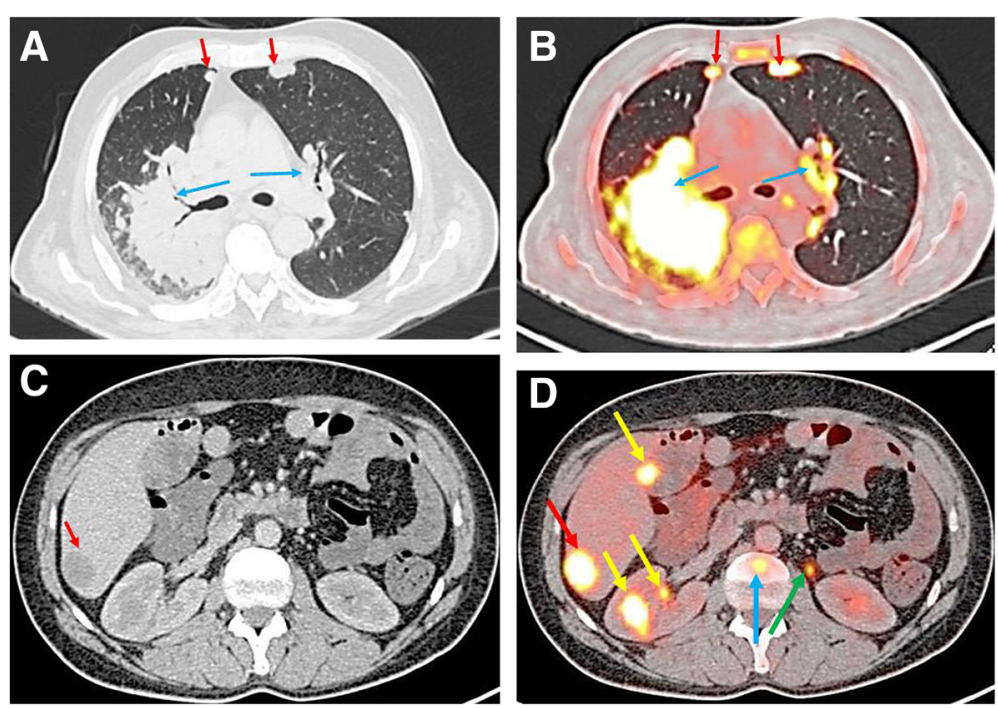

Fig. 15 Male patient 70 years old diagnosed as pulmonary Non- Hodgkin lymphoma which proven by histopathology. a Axial contrast enhanced CT (CECT) of the chest, pulmonary window, showing bilateral hilar masses (blue arrows) which is more sizable on the right side that infiltrates most of the right lung field and bilateral anterior subpleural nodules (red arrows). b Axial PET/CT showing avid FDG uptake of the bilateral hilar masses (blue arrows) and bilateral anterior subpleural nodules (red arrows) previously seen on CECT. c Axial CECT of the abdomen showing single hepatic focal lesion seen at segment VI (red arrow). d Axial PET/CT showing avid FDG uptake of the hepatic focal lesion seen at segment VI (red arrow) previously seen on CECT, with avid FDG uptake of: another focal hepatic lesion seen at segment $V$ (yellow long arrow), two right renal focal lesions (yellow short arrows), focal dorsal vertebral bone marrow lesion (blue arrow), and small left paraaortic lymph node (green arrow), not seen on CECT 

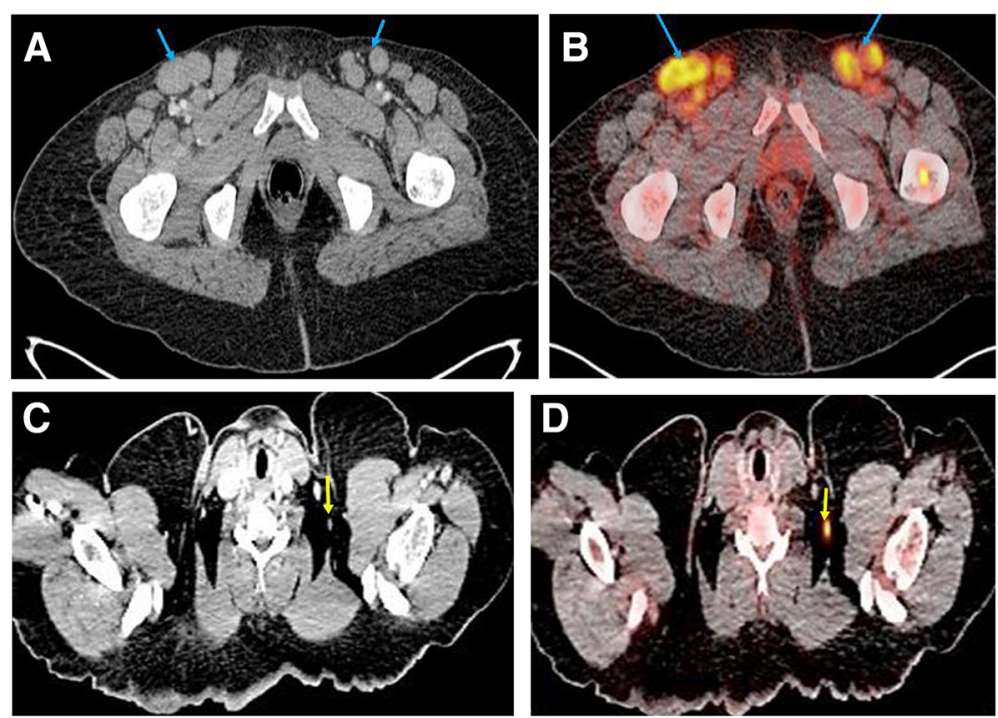

Fig. 16 Male patient 73 years old diagnosed and proven by histopathology as having inguinal lymph nodal non-Hodgkin lymphoma. a Axial contrast enhanced CT (CECT) of the pelvis showing bilateral bulky inguinal lymph nodes (blue arrows). b Axial PET/CT showing avid FDG uptake of the bilateral bulky inguinal lymph nodes (blue arrows) previously seen on CECT. c Axial CECT showing small left cervical lymph node 6 mm in short axis (yellow arrow). d Axial PET/CT showing avid FDG uptake of the small left cervical lymph node (yellow arrow)

\section{Recommendations}

Novel tools such as the use of Raman spectroscopy (RS) enhanced by using a portable fiber-optic probe, i.e., Raman-enhanced spectroscopy (RESpect) probe, could be leveraged to provide rapid and real-time assessment of malignant lesions as suggested by Agsalda-Garcia et al. [25] that will help to overcome the limitation of taking biopsies form suspected lesions in order to define a gold standard.

A future study using the same PET/CT scanner to compare low-dose unenhanced PET/CT and full-dose contrast-enhanced PET/CT in lymphoma assessment is recommended aiming at reducing patient radiation exposure.
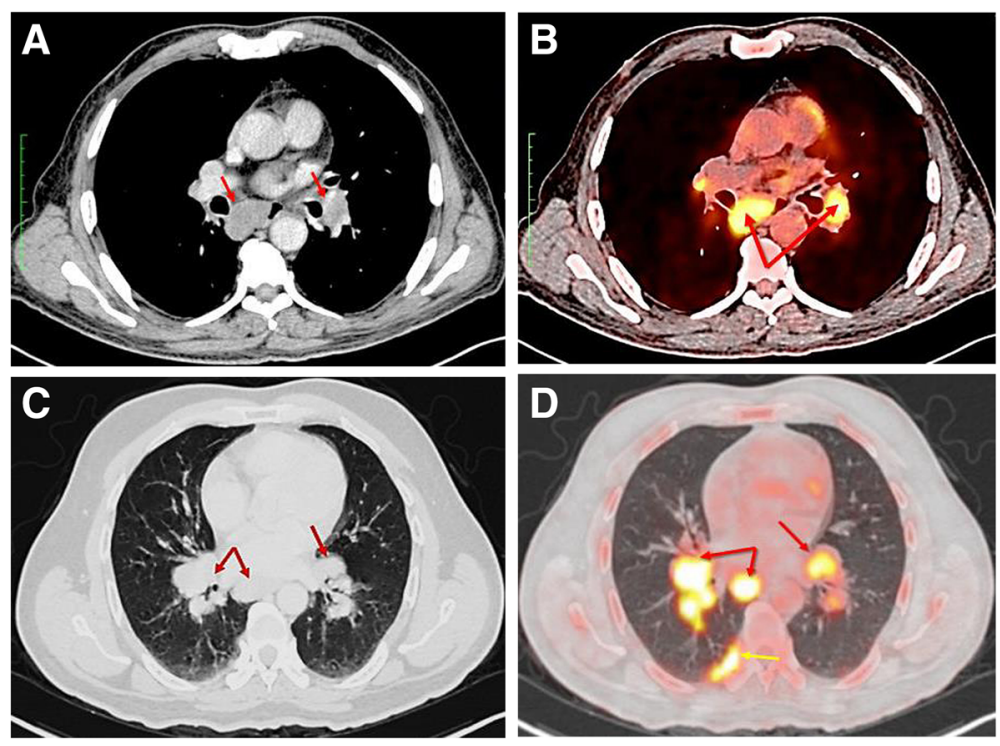

Fig. 17 Male patient 66 years old diagnosed as mediastinal lymph nodal non-Hodgkin lymphoma which proven by histopathology. a Axial contrast enhanced $C T$ (CECT) showing enlarged mediastinal and left hilar lymph nodes (red arrows). b Axial PET/CT showing avid FDG uptake of the enlarged mediastinal and hilar lymph nodes (red arrows) previously seen on CECT. c Axial CECT showing enlarged bilateral hilar and mediastinal lymph nodes (red arrows). d Axial PET/CT showing avid FDG uptake of the enlarged bilateral hilar and mediastinal lymph nodes (red arrows) previously seen on CECT, with avid FDG uptake of a small focal right posterior pleural thickening, hardly recognized on CECT (yellow arrow) 


\section{Abbreviations}

NHL: Non-Hodgkin's lymphoma; HL: Hodgkin's lymphoma; CT: Computed tomography; PET: Positron emission tomography; FDG: 2-[Fluorine-18] fluoro2-deoxy-D glucose; CECT: Contrast-enhanced computed tomography; FDGPET/CT: 2-[Fluorine-18] fluoro-2-deoxy-D-glucose positron emission tomography combined to computed tomography; SUV: Standardized uptake value; IEC: Institutional ethics committee; IV: Intravenous injection; HD: High definition; MRI: Magnetic resonance imaging; RS: Raman spectroscopy; RESpect: Raman-enhanced spectroscopy

\section{Acknowledgement}

I would like to offer special limitless thanks and deep gratitude to the late Prof Dr. Adel Mohamed Elwakeel Professor of Radiodiagnosis, Faculty of Medicine, Menoufia University, Menoufia, Egypt who although no longer with us, continues to inspire by his example and dedication to the students he served over the course of his career.

\section{Authors' contributions}

All the authors share in study conception and design, acquisition of data, analysis and interpretation of data, drafting, and revision of manuscript. All authors have read and approved the manuscript. AAZ, HHM, BAAEM and $\mathrm{MMH}$ 1. Substantial contribution to the conception of the study. 2. Substantial contribution to the design of the study. 3. Substantial contribution to the acquisition, analysis of the data. 4. Substantial contribution to the interpretation of data. 5. Substantial contribution to the creation of the final work. 6. Substantial contribution to the study revision. 7. Substantial contribution to the accuracy or integrity of the submitted manuscript.

\section{Funding}

None.

\section{Availability of data and materials}

The datasets used and/or analyzed during the current study are available from the corresponding author on reasonable request.

\section{Ethics approval and consent to participate}

Our study was approved by ethical and scientific committee - Faculty of Medicine - Menoufia University Ref. No. 86/1//9/2016. Written informed consent form was obtained from every patient after detailed explanation of the study.

\section{Consent for publication}

All patients included in this research gave written informed consent to publish the data contained within this study.

\section{Competing interests}

The authors declare that they have no competing interests.

\section{Author details}

${ }^{1}$ Radiodiagnosis Department, Faculty of Medicine, Menoufia University, Menoufia, Egypt. ${ }^{2}$ Radiology Department, National Liver Institute, Menoufia University, Menoufia, Egypt.

Received: 23 June 2020 Accepted: 14 September 2020

Published online: 23 October 2020

\section{References}

1. D'Sauza M, Jaimini A, Bansal A et al (2013) FDG-PET/CT in lymphoma. IJR 23: 354-365 http://www.ijri.org/text.asp?2013/23/4/354/125626

2. Dhanapathi $\mathrm{H}$ and Kumarg R: F-18 FDG PET/CT in the management of lymphoma, IJO. 2007; 28:17-23. Department of Nuclear Medicine, AlIMS, New Delhi-110029 Correspondence to: RAKESH KUMARE-mail: rkphulia@hotmail.com

3. Johnson S, Kumar A, Matasar M, Schöder H, Rademaker J (2015) Imaging for staging and response assessment in lymphoma, Radiology. IJR 276:323-338. https://doi.org/10.1148/radiol.2015142088

4. Buchmann I, Reinhardt M, Elsner K, et al: 2-(fluorine-18) fluoro-2-deoxy-Dglucose positron emission tomography in the detection and staging of malignant lymphoma. A bicenter trial. Cancer. 2001; 91(5):889-899. inga. buchmann@medizin.uni-ulm.de.
5. Connors J (2015) Risk assessment in the management of newly diagnosed classical Hodgkin lymphoma. Blood 125(11):1693-1702. https://doi.org/10. 1182/blood-2014-07-537480

6. Edwards S, Jacks L, Moskowitz C et al (2010) Stanford V program for locally extensive and advanced Hodgkin lymphoma: the Memorial Sloan-Kettering Cancer Center experience. Ann Oncol 21(3):574-581. https://doi.org/10. 1093/annonc/mdp337

7. Frampas E (2013) Lymphoma: Basic points that radiologists should know, diagnostic and interventional imaging. 94:131-144. https://doi.org/10.1016/j. diii.2012.11.006

8. Buchpiguel C (2011) Current status of PET/CT in the diagnosis and follow up of lymphomas. RBHH 33:140-147. https://doi.org/10.5581/1516-8484. 20110035

9. Chin C, Shi C, Hung T et al (2017) Tumor and bone marrow uptakes on [18F] fluorodeoxyglucose positron emission tomography/computed tomography predict prognosis in patients with diffuse large B-cell lymphoma receiving rituximab-containing chemotherapy. Medicine (Baltimore) 96(45):e8655. https://doi.org/10.1097/MD.0000000000008655

10. Kasamon $Y$, Jones $R$, Wahl $R$ (2007) Integrating PET and PET/CT into the riskadapted therapy of lymphoma. J Nucl Med 48:19-27 120-25

11. Osipov M, Vazhenin A, Kuznetsova A, Aksenova I, Vazhenina D, Sokolnikov M (2020) PET-CT and occupational exposure in oncological patients. 2:2. https://doi.org/10.28991/SciMedJ-2020-0202-3

12. Gomez A, Santana PC, Mourão AP (2020) Dosimetry study in head and neck of anthropomorphic phantoms in computed tomography scans. 2:1. https://doi.org/10.28991/SciMedJ-2020-0201-6

13. Yuhao L, Lisha J, Haitao W et al (2019) Effective radiation dose of 18f-fdg PET/CT: how much does diagnostic CT contribute? Radiat Prot Dosim 187: 183-190. https://doi.org/10.1093/rpd/ncz153

14. Kostakoglu L, Cheson B (2014) Current role of FDG PET/CT in lymphoma. Eur J Nucl Med Mol Imaging 41:1004-1027

15. Raanani $P$, Shasha $Y$, Perry $C$ et al (2006) Is CT scan still necessary for staging in Hodgkin and non-Hodgkin lymphoma patients in the PET/CT era? Ann Oncol 17:117-122. https://doi.org/10.1093/annonc/mdj024

16. Mozaffer R, Sadiqa S (2008) Presentation, staging and diagnosis of lymphoma: a clinical perspective. J Ayub Med Coll Abbottabad 20:4 http://www.ayubmed.edu.pk/JAMC/PAST/20-4/Mozaffer.pdf

17. Roman E, Smith A (2011) Epidemiology of lymphomas. Histopathology 58(1):4-14 10.1111/j.1365-2559.2010.03696.x.hal-00610747 https://hal. archives-ouvertes.fr/hal-00610747

18. Ricard F, Giammarile F, Tychyj-Pinelb C et al (2014) PET-CT and diagnostic CT: the synergy of metabolic and morphological data in onco-haematology. Diag Interv Imaging 95:55-62. https://doi.org/10.1016/j.diii.2013.07.008

19. Rodriguez B, Gomez N, Pinilla I et al (2006) PET/CT in Lymphoma: prospective study of enhanced full-dose PET/CT versus unenhanced lowdose PET/CT. J Nucl Med 47:1643-1648 PMID: 17015900

20. La Fougere C, Hundt W, Brockel N et al (2006) Value of PET/CT versus PET and CT performed as separate investigations in patients with Hodgkin's disease and non-Hodgkin's lymphoma. Eur J Nucl Med Mol Imaging 33: 1417-1425. https://doi.org/10.1007/s00259-006-0171-x

21. Kwee $T$, Kwee R, Nievelstein $R$ (2008) Imaging in staging of malignant lymphoma: a systematic review. Blood 111:504-516. https://doi.org/10.1182/ blood-2007-07-101899

22. De Jong P, Van Ufford $\mathrm{H}$, Baarslag $\mathrm{H}$ et al (2009) $\mathrm{CT}$ and $18 \mathrm{~F}-\mathrm{FDG}$ PET for noninvasive detection of splenic involvement in patients with malignant lymphoma. AJR Am J Roentgenol 192(3):745-753

23. Othman A, Nasr M, Abdel-Kawi M (2019) Beyond lymph nodes: 18 F-FDG $\mathrm{PET} / \mathrm{CT}$ in detection of unusual sites of extranodal lymphoma. Egypt $J$ Radiol Nucl Med 50(1):29

24. Luminari S, Biasoli I, Arcaini L, Versari A, Rusconi C, Merli F et al (2013) The use of FDG-PET in the initial staging of 142 patients with follicular lymphoma: A retrospective study from the FOLL05 randomized trial. Ann Oncol 24(8):2108-2112. https://doi.org/10.1093/annonc/mdt137

25. Agsalda-Garcia M, Shieh T, Souza R et al (2020) Raman-enhanced spectroscopy (RESpect) probe for childhood non-Hodgkin lymphoma. 2:1. https://doi.org/10.28991/SciMedJ-2020-02011

\section{Publisher's Note}

Springer Nature remains neutral with regard to jurisdictional claims in published maps and institutional affiliations. 